\title{
Model-Independent Structure Determination of the $\operatorname{InSb(111)} 2 \times 2$ Surface with Use of Synchrotron X-Ray Diffraction
}

\author{
J. Bohr, ${ }^{(a)}$ R. Feidenhans'l, M. Nielsen, and M. Toney ${ }^{(\mathrm{b})}$ \\ Riso National Laboratory, DK-4000 Roskilde, Denmark \\ and \\ R. L. Johnson \\ Max Planck Institut für Festkörperforschung, D-7000 Stuttgart, Federal Republic of Germany \\ and \\ I. K. Robinson \\ AT\&T Bell Laboratories, Murray Hill, New Jersey 07974 \\ (Received 26 December 1984)
}

\begin{abstract}
We have measured accurate $x$-ray crystallographic data from the $\operatorname{InSb}(111) 2 \times 2$ reconstructed surface using synchrotron radiation from the DORIS storage ring at DESY. We have analyzed these independently of all models and find a structure with seven atoms in the unit cell, implying that one site is unoccupied. The bonding configuration is reasonable for a III-V semiconductor surface, and is topologically identical (in projection) to the "vacancy-buckling" model proposed for the GaAs(111) $2 \times 2$ surface. The differences between the InSb and GaAs structures are significant and reflect chemical trends in the periodic table.
\end{abstract}

PACS numbers: $68.20 .+\mathrm{t}, 61.10 . \mathrm{Fr}$

Orbital rehybridization is currently considered to be a major determinant of the atomic geometry in compound-semiconductor surfaces. The III-V(110) surfaces, well understood from low-energy electrondiffraction (LEED) studies, ${ }^{1}$ buckle under this influence to reduce the bond angles at the surface group-V element and increase those at the group-III element, demonstrating $p$-type and $s p^{2}$-type bonding tendencies, respectively. ${ }^{2}$ A buckling model has also been proposed by Haneman ${ }^{3}$ to allow similar rehybridization in the reconstructed GaAs(111) $2 \times 2$ surface, but $\mathrm{Chadi}^{4}$ has shown that the buckled arrangement is unstable to the removal of one surface $\mathrm{Ga}$ atom. The vacancy soformed has been identified in a recent LEED structure analysis of $\mathrm{GaAs}(111),{ }^{5}$ which shows dramatic inplane buckling of the remaining seven atoms in the surface unit cell. Because glancing-incidence $\mathrm{x}$-ray diffraction is highly sensitive to in-plane surface structures, ${ }^{6}$ it is well-suited to this problem, and so we applied it to the InSb(111) $2 \times 2$ reconstructed surface and determined the atomic arrangement. Our analysis includes no prior knowledge of these models and derives directly a seven-atom structure of the projected unit cell. We are able even to identify the atom types and conclude that it is an In atom missing, implicating "vacancy buckling" in $\operatorname{InSb}(111)$ also.

Precisely cut, oriented, and polished InSb crystals were cleaned in $2 \times 10^{-10}$ mbar by repeated cycles of sputtering with $500-\mathrm{eV} \mathrm{Ar}^{+}$ions and annealing at $420^{\circ} \mathrm{C}$ for several hours. The (111) surface gave a sharp $2 \times 2$ LEED pattern, while the (111) surface was $3 \times 3$; the latter is discussed elsewhere and not considered further here. ${ }^{7}$ Valence-band energy distribu- tions curves were recorded at $h \nu=30 \mathrm{eV}$ with use of synchrotron light from the storage ring DORIS at Deutsches Elektronen-Synchrotron in Hamburg; these were found to be much more sensitive to surface contamination than conventional Auger spectra, and showed the surface to be clean. The sample was then transferred in UHV to a small, ion-pumped diffraction cell with a $360^{\circ}$ beryllium window and was mounted on a diffractometer on the adjacent beam line.

The white synchrotron beam was vertically monochromated by parallel $\mathrm{Ge}(111)$ crystals and filtered by total reflection from a horizontal flat Au-coated mirrror. The diffraction took place in the horizontal plane of an air table which was tilted with respect to the beam to control the angle of incidence on the sample. The natural vertical collimation of the source and the horizontal geometry thus confined the entire range of incident $x$ rays to within $\pm 0.005^{\circ}$ of the critical angle for $\mathrm{InSb}$ (determined to be $0.236^{\circ}$ ) throughout the experiment. In-plane collimation was provided by 1 $\mathrm{mm}$ slits before and after the sample which kept the sensitive area of the diffractometer within the sample borders. The wavelength $(\lambda=1.119 \AA)$ was chosen short to minimize polarization effects in the outermost reflections.

Half-order surface Bragg reflections were collected with a position-sensitive detector set vertical to accept a $4^{\circ}$ range of exit angles. Scans of the azimuthal angle $\omega$ were integrated and background subtracted for every reflection. Rod profiles, accumulated in the positionsensitive detector, were flat except for a sharp edge and slight enhancement at the critical angle of exit from the surface; we therefore concluded that the dif- 
fraction arose from a single plane of atoms and used the total intensity under each rod measured. Thirtyeight intensities were reduced to sixteen by symmetry equivalence; the reproducibility gave us their uncertainty. The values were corrected for polarization $\left(\cos ^{2} 2 \theta\right)$, Lorentz factor $\left[(\sin 2 \theta)^{-1}\right]$, and variation of active sample area $\left[(\sin 2 \theta)^{-1}\right]$. Structure-factor amplitudes were derived and are listed in Table I. The maximum count rate was $30 \mathrm{sec}^{-1}$ under "parasitic" running conditions $(5 \mathrm{GeV}, 40 \mathrm{~mA})$. A standard reflection was measured repeatedly over the $33 \mathrm{~h}$ of data collection and seen to remain constant, suggesting that surface deterioration was small. A second set of data, in which the ambient cell pressure was higher, was found to have exponential loss of intensity, with a half-life of $23 \mathrm{~h}$; after rescaling, these data were in good agreement.

Figure 1(a) shows the pair-correlation (Patterson) function calculated from the observed structure factors in Table I,

$$
P(x, y)=\sum_{h k}\left|F_{h k}^{\text {obs }}\right|^{2} \cos 2 \pi(h x+k y) .
$$

There are three clear nonorigin peaks that must correspond to interatomic vectors in the surface structure. The bulk structure projected into the (111) plane is a

honeycomb arrangement of atoms, in which the repeating unit is a covalently bonded hexagon, shown as open circles in Fig. 1(b). If the hexagon is placed in a $2 \times 2$ unit cell, interatomic vectors are the open circles shown in Fig. 1(c). If the hexagon is distorted so as to conserve bond length, as the closed circles in Fig. 1(b) indicate, the peaks in the pair-correlation function move to the positions indicated in Fig. 1(c), which are exactly those observed in Fig. 1(a). This distorted hexagon is the only simple arrangement of atoms with $3 m$ symmetry that agrees with the Patterson function.

A structure-factor calculation was then performed with these six atoms in the $2 \times 2$ unit cell at coordinates $\left(x_{j}, y_{j}\right)$ and form factors $f_{j}\left(q_{h k}\right)$,

$$
\begin{aligned}
& \left|F_{h k}^{\mathrm{calc}}\right| \exp \left(i \alpha_{h k}^{\mathrm{calc}}\right) \\
& \quad=\sum_{j} f_{j}\left(q_{h k}\right) \exp \left[2 \pi i\left(h x_{j}+k y_{j}\right)\right] .
\end{aligned}
$$

When a comparison of the $\left|F_{h k}^{\text {calc }}\right|$ with the observed $F_{h k}^{\text {obs }}$ (Table I) is made, however, the agreement is poor. The mean-square residual is $\chi^{2}=125$, indicating that a typical calculated amplitude is more than ten standard deviations away from the observed value. Missing parts of the structure are revealed in Fig. 2 by a map of the difference electron density,

$$
\Delta \rho(x, y)=\sum_{h k}\left(F_{h k}^{\text {obs }}-\left|F_{h k}^{\text {calc }}\right|\right) \exp \left\{i\left[\alpha_{h k}^{\text {calc }}-2 \pi(h x+k y)\right]\right\} .
$$

A single peak, well above the noise level of the map, indicates the discrepancy: a seventh atom sitting on one of the threefold axes is required to complete the structure. The seven-atom structure has a residual of $\chi^{2}=25$ which

TABLE I. Observed and calculated surface structure factors for $\operatorname{InSb}(111) 2 \times 2$. The indices $(h, k)$ used to label the reflections refer to the hexagonal coordinate frame defined in Fig. 2. The superscript 6 refers to the values calculated for the six-atom model derived directly from the Patterson function after adjustment of a scale factor only. The superscript 7 refers to the final four-parameter model.

\begin{tabular}{rrrrr}
\hline \hline \multicolumn{1}{c}{$h$} & \multicolumn{1}{c}{$F_{h k}^{\text {obs }}$} & \multicolumn{1}{c}{$F_{h k}^{\text {calc }^{6}}$} & $F_{h k}^{\text {calc }}$ \\
\hline $1 / 2$ & 0 & $3.5 \pm 0.2$ & 9.0 & 4.0 \\
$1 / 2$ & $1 / 2$ & $6.2 \pm 0.5$ & 9.4 & 6.4 \\
1 & $1 / 2$ & $21.1 \pm 0.4$ & 10.0 & 21.0 \\
$3 / 2$ & 0 & $32.1 \pm 0.6$ & 30.0 & 31.6 \\
$3 / 2$ & $1 / 2$ & $8.6 \pm 0.5$ & 3.1 & 9.0 \\
$3 / 2$ & 1 & $7.6 \pm 0.5$ & 13.8 & 8.0 \\
$3 / 2$ & $3 / 2$ & $9.3 \pm 1.0$ & 11.3 & 11.6 \\
2 & $1 / 2$ & $18.5 \pm 0.9$ & 15.9 & 19.0 \\
2 & $3 / 2$ & $17.5 \pm 0.8$ & 9.3 & 17.3 \\
$5 / 2$ & 0 & $26.2 \pm 1.1$ & 14.6 & 22.0 \\
$5 / 2$ & $1 / 2$ & $16.6 \pm 1.0$ & 5.2 & 17.5 \\
$5 / 2$ & 1 & $29.4 \pm 1.2$ & 26.5 & 29.2 \\
$5 / 2$ & $3 / 2$ & $14.1 \pm 0.8$ & 9.0 & 14.7 \\
3 & $1 / 2$ & $3.8 \pm 2.6$ & 4.1 & 2.6 \\
$7 / 2$ & 0 & $8.9 \pm 2.2$ & 12.2 & 9.1 \\
$7 / 2$ & $1 / 2$ & $12.8 \pm 2.0$ & 12.6 & 13.7 \\
\hline \hline
\end{tabular}
drops to 2.1 when the positions of the atoms are leastsquares refined (consistent with the symmetry) and a

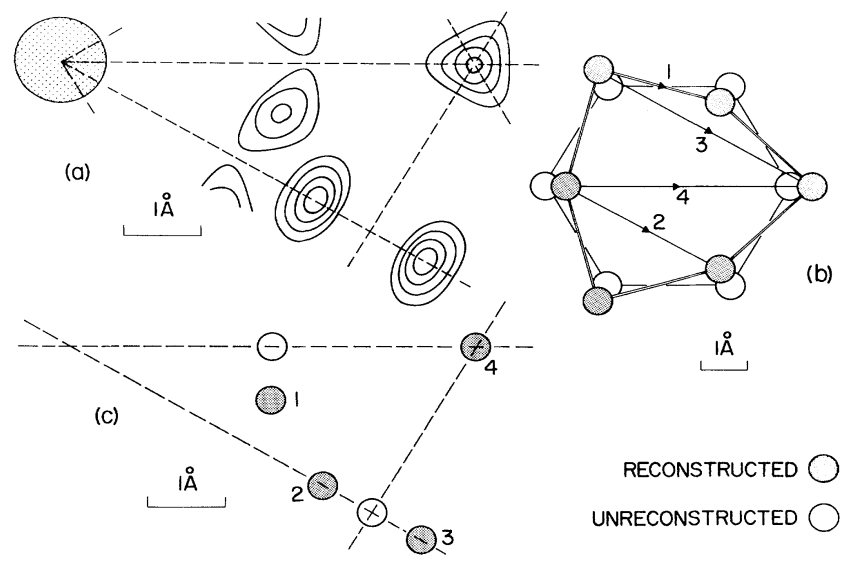

FIG. 1. (a) Repeating unit of the Patterson function calculated for the $F_{h k}^{\text {obs }}$ in Table I. Positive contour levels above zero are shown. Dashed mirror lines surround the asymmetric repeating unit. The shaded circle is the origin peak that rises seventeen contour levels. (b) Distortion of a hexagonal arrangement of atoms taken from the projected unreconstructed InSb(111) surface. (c) Pair-correlation peaks 1 to 4 derived from vectors 1 to 4 in (b). 


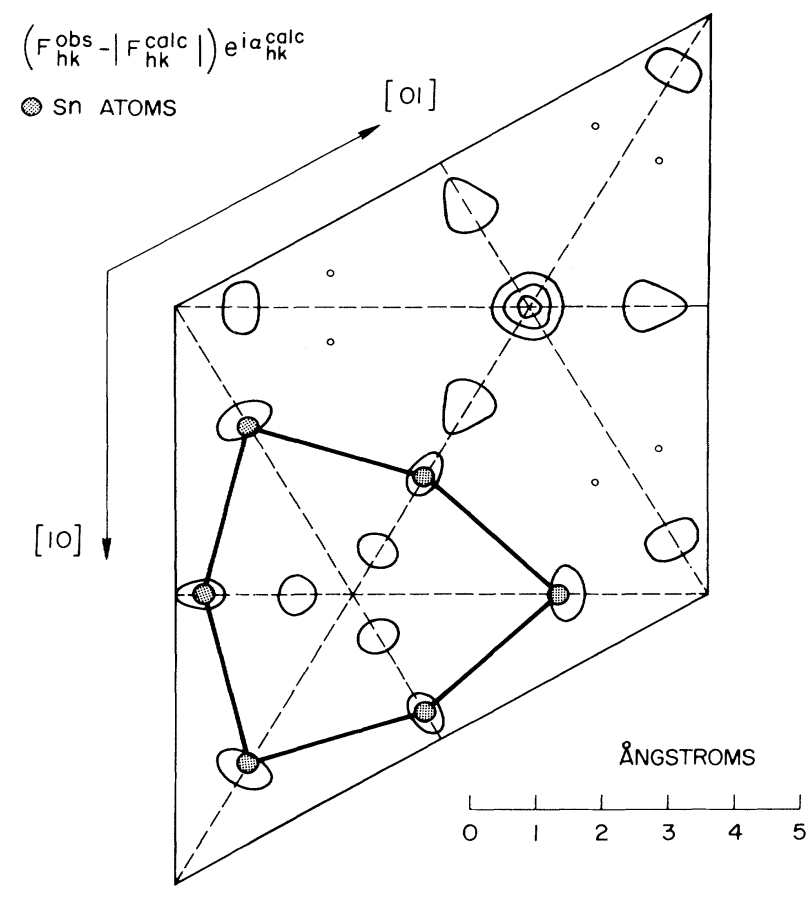

FIG. 2. Difference Fourier map of one $\operatorname{InSb}(111) 2 \times 2$ unit cell. Positive contours above zero are shown. The hexagonal unit-cell vectors shown are used throughout the paper and defined as follows: $[1,0]_{\text {hex }}=\left[\frac{1}{2},-\frac{1}{2}, 0\right]_{\text {bulk }}$ and $[0,1]_{\text {hex }}=\left[0, \frac{1}{2},-\frac{1}{2}\right]_{\text {bulk }}$.

thermal-vibration parameter is included. At this stage the calculated amplitudes are only slightly outside the error bars of the observations. Repeating the difference-map calculation [Eqs. (1) and (2)] with the new calculated structure factors generates only noise. Furthermore, testing of models with one less or one more atom, even with allowance of parameter relaxation by least-squares refinement, always gives residuals in excess of 30 .

The analysis above has not differentiated between the In $(Z=49)$ and $\mathrm{Sb}(Z=51)$ atom types; form factors for $\operatorname{Sn}(Z=50)$ were used throughout the calcalculations. When the atomic number of the outer three atoms (symmetry equivalent) of the distorted hexagon is allowed to vary (with the others fixed at 50 ), the best agreement is for $Z_{1}=54.6$; for the inner three, $Z_{2}=48.5$; for the seventh atom on the threefold axis, $Z_{3}=50.3$. Clear assignments of $Z_{1}=51(\mathrm{Sb})$ and $Z_{2}=49$ (In) can be made, with a probable assignment of $Z_{3}=51(\mathrm{Sb})$. The last statement is strengthened by the observation that the assignment means every $\mathrm{Sb}$ has only In nearest neighbors in the plane and vice versa. Furthermore, since this structure has three In and four $\mathrm{Sb}$ atoms in the $2 \times 2$ unit cell, we are seeing the projection of a single layer of InSb with a vacant In site.
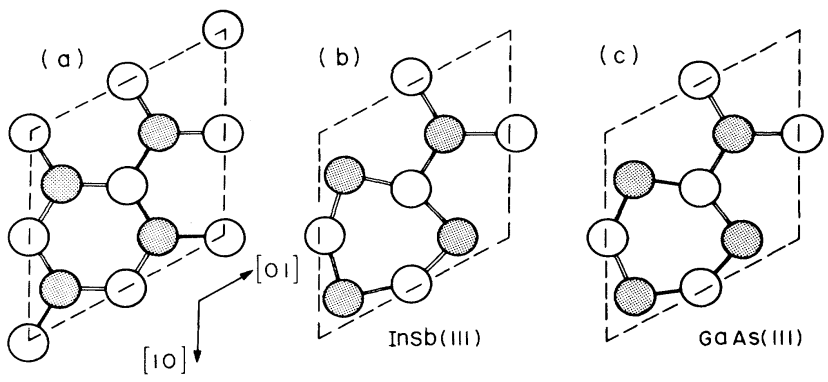

FIG. 3. Scale drawing of the projected atomic positions in (a) an unreconstructed III-V(111) surface, (b) InSb(111)2 $\times 2$, (c) GaAs(111) $2 \times 2$ (Ref. 5). Group-V atoms are shaded.

In our two-dimensional determination of the InSb structure we cannot say which layer reconstructs, but when we consider its remarkable similarity with the GaAs(111) $2 \times 2$ structure determined by LEED, ${ }^{5}$ we can be confident that it is the top layer. Drawn to scale in Fig. 3 are the $\operatorname{InSb}(111) 2 \times 2$ reconstructed surface, the GaAs(111) $2 \times 2$ surface (lattice-parameter adjusted), and an ideal unreconstructed InSb(111) surface. We have assigned bonds between the atoms as shown and connected the reconstructed layer to the bulk in exactly the same manner as GaAs, although the assignment with the layer rotated $180^{\circ}$ (i.e., with a stacking fault between the first and second double layers) is strictly indistinguishable. The final model for InSb is obtained in a four-parameter least-squares fit: There is an arbitrary scale factor, an overall Debye-Waller thermal parameter of $B=1.1 \pm 1.1 \AA^{2}$ for all atoms, a radial outward displacement of the three equivalent $\mathrm{Sb}$ atoms of $0.45 \pm 0.04 \AA$, and a radial inward displacement of $0.23 \pm 0.05 \AA$ for the three In atoms. The residual has dropped to $\chi^{2}=1.8$ with the correct assignment of atoms. The corresponding displacements for GaAs are $0.28 \AA$ for As and $0.10 \AA$ for Ga from LEED experiments. ${ }^{5}$ The total-energy - minimization calculation of Chadi gives very similar values of 0.30 and $0.15 \AA$, respectively, for As and $\mathrm{Ga}^{4}$ The thermal parameter corresponds to a onecomponent vibration amplitude of $0.12 \AA$, which compares favorably with the value of $0.13 \AA$ for bulk InSb. ${ }^{8}$

Visual inspection of Fig. 3 and detailed analysis of the structural parameters in Table II show a clear trend from $\mathrm{GaAs}$ to InSb. The LEED analysis ${ }^{5}$ shows the surface layer of GaAs(111) to be extremely flat, within 0.2 A: The bond lengths and angles are well approximated by their values in projection onto the surface plane, to within $0.01 \AA$ and $0.2^{\circ}$, respectively. If the InSb(111) surface is correspondingly flat, then the projected bond lengths and angles derived in Table II are equally significant. The surface bond lengths are found to be very close to the bulk values in both cases; 
TABLE II. Derived structure parameters for InSb(111) and comparison with GaAs(111). Atom $A$ refers to the three group-III elements (Ga or In), atom $B_{1}$ refers to the three group-V elements (As or $\mathrm{Sb}$ ) in the "hexagon," and $B_{2}$ refers to the lone group $-\mathrm{V}$ element on the threefold axis (see Fig. 3).

\begin{tabular}{lccc}
\hline \multicolumn{1}{c}{ Parameter } & InSb(111) & \multicolumn{2}{c}{ GaAs(111) } \\
& This work & Ref. 5 & Ref. 4 \\
\hline$B_{1}$ bond angle & $96 \pm 2^{\circ}$ & $104^{\circ}$ & $102^{\circ}$ \\
$A$ bond angles & $144 \pm 2^{\circ}$ & $136^{\circ}$ & $138^{\circ}$ \\
& $108 \pm 2^{\circ}$ & $112^{\circ}$ & $111^{\circ}$ \\
$A B_{1}$ bond length & $2.82 \pm 0.5 \AA$ & $2.42 \AA$ & $2.41 \AA$ \\
$A B_{2}$ bond length & $2.87 \pm 0.5 \AA$ & $2.41 \AA$ & $2.46 \AA$ \\
Bulk bond length & $2.81 \AA$ & \multicolumn{2}{c}{$2.45 \AA$} \\
\hline \hline
\end{tabular}

the fact that the bonds are very slightly longer than the bulk not only confirms that the surface is flat but also rules out any possibility of $\pi$ bonding between the surface atoms. The trend in bond angle from As to $\mathrm{Sb}$ reflects the increasing tendency down the group-V elements to form $p$-type bonds $\left(90^{\circ}\right.$ angle) instead of $s p^{3}$ $\left(109^{\circ}\right.$ angle). The observed bond angles at the trivalent surface $\mathrm{Sb}$ and $\mathrm{As}$ are close to the ideal values found in simple compounds: $\mathrm{SbBr}_{3}$ has an angle of 95.; $\mathrm{AsBr}_{3}$ has $100^{\circ} .{ }^{\circ}$ The bond angles for the trivalent surface In atoms are less close to the ideal $120^{\circ}$ for $s p^{2}$ bonding. This may imply that the electronic rehybridization is less complete for In than $\mathrm{Sb}$, but may be simply a consequence of the $s p^{2}$ bond angles being less stiff than those of the $p$ type.

In summary, we have presented the most accurate $\mathrm{x}$-ray surface crystallographic data measured to date and demonstrated that a surface structure can be derived without reference to models. Useful stereochemical data have been obtained, even though all the measurements considered were strictly twodimensional and so resulted in a projection of the surface atomic arrangement.

We wish to thank Hamburger Synchrotronstrahlungslabor for hospitality and assistance and $M$. Schulter for helpful discussion. This work was supported in part by the Danish Natural Science Foundation and by NATO.

(a) Present address: Brookhaven National Laboratory, Upton, N.Y. 11973.

(b) Permanent address: IBM Research Laboratory, San Jose, Cal. 95193.

${ }^{1}$ A. R. Lubinsky, C. B. Duke, B. W. Lee, and P. Mark, Phys. Rev. Lett. 36, 1058 (1976); S. Y. Tong, A. R. Lubinsky, B. J. Mrstik, and M. A. van Hove, Phys. Rev. B 17, 3303 (1978)

2D. J. Chadi, Phys. Rev. Lett. 41, 1062 (1978).

${ }^{3}$ D. Haneman, Phys. Rev. 121, 1093 (1961).

${ }^{4}$ D. J. Chadi, Phys. Rev. Lett. 52, 1911 (1984).

${ }^{5}$ S. Y. Tong, G. Xu, and W. N. Mei, Phys. Rev. Lett. 52, 1693 (1984).

6I. K. Robinson, Phys. Rev. Lett. 50, 1145 (1983);

P. Eisenberger and W. C. Marra, Phys. Rev. Lett. 46, 1081 (1981).

${ }^{7}$ R. L. Johnson, J. H. Fock, I. K. Robinson, J. Bohr, R. Feidenhans'1, J. Als-Nielsen, M. Nielsen, and M. Toney, in Proceedings of the First International Conference on Structure of Surfaces, Berkeley, 13-16 August 1984 (to be published).

${ }^{8}$ R. N. Kyutt, Fiz. Tverd. Tela (Leningrad) 20, 395 (1978) [Sov. Phys. Solid State 20, 227 (1978)].

${ }^{9}$ A. F. Wells, Structural Inorganic Chemistry (Clarendon, Oxford, 1975). 